\title{
Skewness as means for separating crackle from screech
}

\author{
Jyothi N. Punekar* and Eldad J. Avital ${ }^{\dagger}$ \\ School of Engineering and Materials Science, Queen Mary University of London, Mile End Road, London, E1 4NS, UK \\ Aldo Rona \\ Department of Engineering, University of Leicester, University Road, Leicester, LE1 7RH, UK
}

\begin{abstract}
Screech and crackle are investigated by far-field acoustic measurements and visualizations of a cold jet from a Mach 2 convergent-divergent nozzle operated over-expanded, fully expanded, and under-expanded. The skewness of the sampled far-field pressure frequency distribution is evaluated at polar angles below $90^{\circ}$. It is found that skewness alone is insufficient for singling out crackling noise, however, a better characterization is obtained by jointly considering the skewness of the acoustic pressure time derivative. New evidence is provided of crackle and screech being competitive mechanisms over the nozzle pressure ratio range 4.3 to 10 . These new physical insights advance the current understanding of high-speed jet aeroacoustics, towards enabling sustainable airport operations of supersonic civil aircraft.
\end{abstract}

\section{Nomenclature}

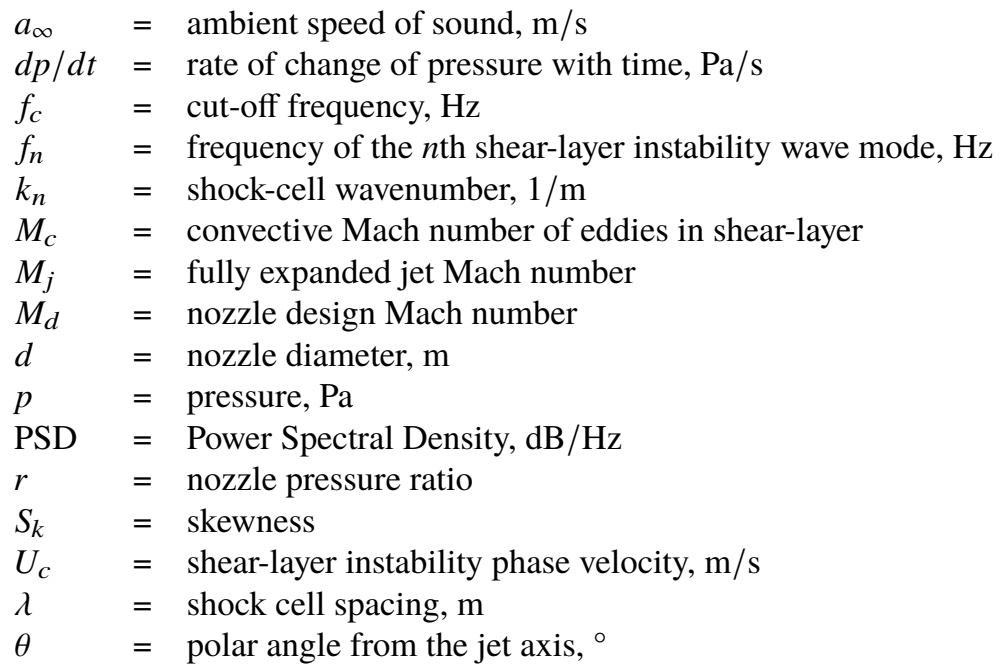

\section{Introduction}

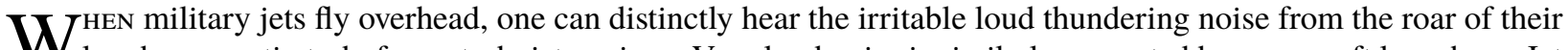
W low-bypass ratio turbofan or turbojet engines. Very loud noise is similarly generated by spacecraft launchers. Jet engines with such high levels of thundering noise can be a concern for the integrity of extensively glazed buildings. The powerful sound field created is also a noise pollution issue, constituting an environmental problem as well as a serious health threat. Although fighter jets tend to avoid overflying densely populated areas, like cities, it has been reported that the noise impairs people's ability to work, study in school, sleep, and consequently has a serious impact on the economy. Engine noise reductions for high speed transport jets has been a major concern in Aeroacoustics. Both theoretical and new computational approaches in conjunction with experimental measurements have been employed to mitigate jet

\footnotetext{
*Visiting Postdoctoral Researcher, School of Engineering and Materials Science, Queen Mary University of London.

${ }^{\dagger}$ Reader, School of Engineering and Materials Science, Queen Mary University of London, AIAA Senior Member

$\doteqdot$ Associate Professor, Department of Engineering, University of Leicester, aldo.rona@le.ac.uk, AIAA Member.
} 
noise and meet international legislation on air transport operation, coordinated by ICAO, the FAA in the US, and the CAA in the UK.

Minimizing the level of sonic boom annoyance for relatively high Mach number cruise to near acceptable limits has become a popular topic in aeroacoustics in recent years, owing to a renewed interest in supersonic business jets. This however leaves the fundamental question of producing acceptable jet noise levels at take-off and at landing, which are likely to take place at airports near major urban centres. This requirement motivates further research aimed at tackling fundamental aspects of high speeds jet noise. At supersonic nozzle exit Mach numbers, the emitted acoustic power output is about 0.01 of the supersonic jet power [1]. However, due to the high directivity of the radiated noise, the radiated noise pollution becomes unbearably annoying, to the point of becoming a serious health hazard. The intense near-field pressure fluctuation can be at a level that it can cause damage to the airframe, specifically to the nozzle and to thin-walled trailing edge aircraft control surfaces. Sonic fatigue thereby becomes a concern for high specific thrust applications in space, defense, and future hypersonic flight technology.

Crackle is a well-known annoying component of supersonic jet turbulent mixing noise. Crackling noise is generated by supersonic eddies and it is found mainly along the Mach wave direction, at a downstream angle $\theta$, which is given by $\cos \theta=M_{c}^{-1}$, where $M_{c}$ is the convective Mach number of the eddies. Crackle has been described by Ffowcs Williams et al. [2] to be made of sudden spasmodic bursts of sound. It is a distinctive aspect of jet noise for its annoyance. It exhibits random bursts of compression sound waves followed by shallow expansion waves. Crackle accounts for approximately 30\% of the total annoyance of jet noise. Punekar et al. [3, 4], at the Queen Mary University of London (QMUL) model jet facility, used contoured nozzles of design Mach number $M_{d}=1.3$ and 2 to show that crackle is present at shallow polar angles from the jet axis. This radiation direction was characterized by a high skewness of the acoustic pressure.

Screech noise arises in incorrectly expanded jets, where a train of shock and expansion waves form, typically in the form of diamond-shaped shock cells. This spatially periodic pattern of compressions and expansions interact with convective shear-layer instabilities, creating a feed-back loop, similar to an edge tone, as described by Powell [5]. The feed-back locks the hydrodynamic and the acoustic fields in a self-sustained resonance that is tonal. This narrow-band frequency characteristics was numerically investigated by time-marching unsteady Reynolds Averaged Navier-Stokes (URANS) simulations for the case of over-expanded axisymmetric jets in [6, 7]. Screech tones, by being typically above the broad-band noise level, have the potential of being perceived as highly annoying. Due to its upstream-propagating component of the feed-back loop, significant noise radiation in screech occurs in the opposite direction to the jet bulk velocity at the fundamental tone, however harmonics can be found in the sideline and downstream of the jet. Punekar et al. [3] also studied the non-linearity of screech and found that screech, although of high amplitude, is not as non-linear as crackle, particularly when considering the acoustic pressure derivative.

A fundamental issue in high speed jet noise is the ability to identify and set apart the different contributions from screech and crackle, so that methods of noise mitigation acting on a specific noise source mechanism can be pursued. The aim of this study is to advance the state of the art in characterizing screech and crackle, based on considerations of skewness of their signals. The impact potential from this fundamental study is significant. It may enable the design of propulsion systems for new supersonic transport (SST) civil aircraft that may operate with an acceptable noise signature.

\section{Experimental set-up}

Fundamental aspects of supersonic jet noise are experimentally investigated using the acoustic chamber at the Queen Mary University of London (QMUL). A schematic of the chamber is shown in Fig. 11 It consists of a $3.5 \mathrm{~m} \times 3.5 \mathrm{~m} \times 2.5 \mathrm{~m}$ high enclosure, wall to wall. The six walls are tiled by $300 \mathrm{~mm} \times 300 \mathrm{~mm} \times 430 \mathrm{~mm}$ tall acoustic foam wedges. The wedges are designed to produce a lower cut-off frequency of $200 \mathrm{~Hz}$. All flat and non-porous acoustically reflective surfaces within the chamber, such as the exhaust funnel and the microphone support beam, are covered in acoustic foam sheets. Internally, the chamber has wedge to wedge dimensions of $2.5 \mathrm{~m} \times 2.5 \mathrm{~m} \times 2.0 \mathrm{~m}$, giving an approximate working floor space of $6 \mathrm{~m}^{2}$.

In this work, this facility is used to test a model scale cold supersonic jet, first over-expanded, then fully expanded, and finally under-expanded. Desiccated compressed air at 13.79 bar gauge (200 psi gauge) from a large volume central storage facility is regulated through a servo-actuated gate valve through a silencer into the nozzle. A convergent-divergent axisymmetric nozzle with a nozzle outlet design Mach number $M_{d}=2$ is used. The nozzle exit diameter is $3 \mathrm{~cm}$. The nozzle is operated over the nozzle pressure ratio range $4.2 \leq r \leq 10$, to measure crackle and screech at both the over-expanded regime and at the under-expanded regime. The fully expanded jet is also characterized at the nozzle pressure ratio $r=7.8$. 


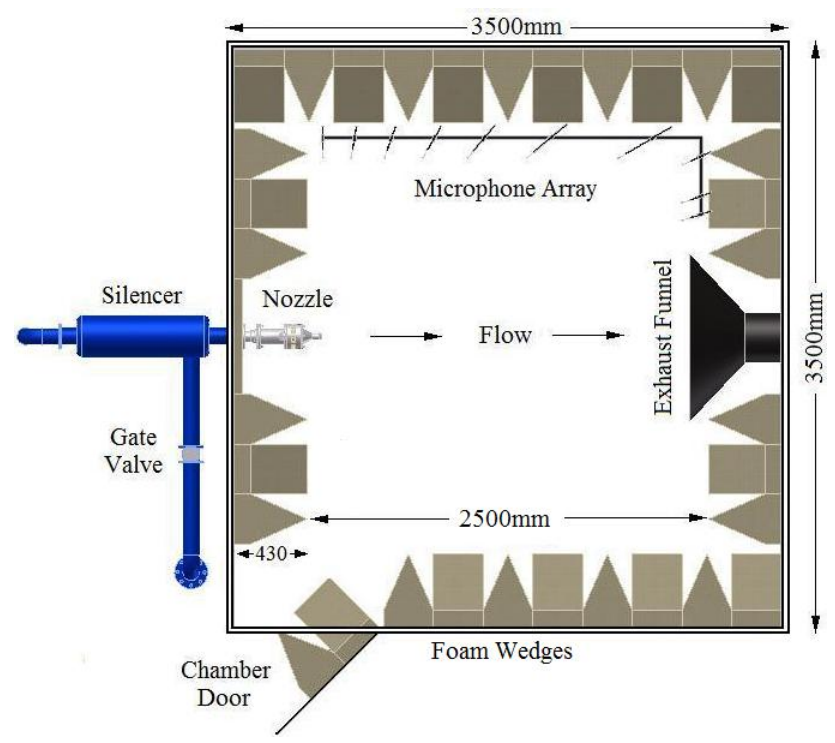

Fig. 1 Schematic of QMUL acoustic chamber.

The nozzle outlet and its near-field discharge are visualized by conventional white light continuous schlieren, across the over-expanded jet regime, over the nozzle pressure ratio range $4.2 \leq r \leq 5.75$. For each nozzle pressure ratio, about $6 \mathrm{~s}$ of digital video footage at 25 frame/s is recorded, from which still frames are extracted to illustrate the jet mean flow. Since the video frame rate is about two orders of magnitude lower than the typical shear-layer instability mode frequencies at this flow regime, the still frames effectively document the time-averaged jet plume.

Measurements are performed using a far-field array of 10 quarter-inch B\&K microphones that is laid out in an $\mathrm{L}$ shape as shown in Fig. 1 in the horizontal plane of the nozzle axis. The amplified microphone signals are conditioned through an anti-aliasing analogue filter with a cut-off frequency $f_{c}$ of $60 \mathrm{kHz}$. The power spectral density of the filtered signal displays a roll-off rate of $102 \mathrm{~dB} /$ decade above $f_{c}$. The analogue to digital conversion of the microphone signals is then performed by a National Instruments acquisition card, at a sampling rate of $131072 \mathrm{~Hz}$. The digitized noise signals are post-processed in Matlab by the Welch periodogram method, using rectangular windows of 1024 samples, $50 \%$ data overlap, and 1024 frequency lines.

The digitized noise samples are then used to generate the normalized frequency plot of the sound pressure, from which the empirical probability density function for the acoustic pressure fluctuation is obtained, for each microphone. This is hereafter referred to as the probability density function of $p$. The skewness $S_{k}(p)$, or third moment, of the probability density function is quantified, which is relevant to the study of crackle.

\section{Results}

\section{A. Jet flow visualization}

Running the $M_{d}=2$ nozzle over-expanded, at a nozzle pressure ratio of approximately $r=4.2$, produces the time-averaged jet flow pattern shown in Fig. 2. The flow runs from left to right, issuing from the black nozzle on the far left. The over-expanded flow is re-compressed by a shock cone that stems from the nozzle exit inner lip. The shock cone is the brightest object in Fig. 2 This compression converges the outflow towards the nozzle axis, as shown by the convergent trajectory of the shear layer that sheds from the nozzle lip. Close to the nozzle axis, the apex of the shock cone is truncated by a Mach disk, which provides a stronger, normal-shock type compression. This Mach disk is a feature of heavily over-expanded $M_{d}=2$ jets also reported in Seiner and Norum [8]. Past the Mach disk, the flow is above ambient pressure and therefore starts expanding through an expansion wave that is shown in the schlieren record as a dark conical region that precedes a further shock cone. This sequence of expansions and compressions repeats in the axial direction, forming a diamond-shaped shock-cell pattern.

Increasing the nozzle pressure ratio to $r=4.7$ avoids the formation of the Mach disk close to the nozzle exit, as shown by the schlieren record in Fig. 3 . This shifts the shock-expansion train axially downstream. As the nozzle 


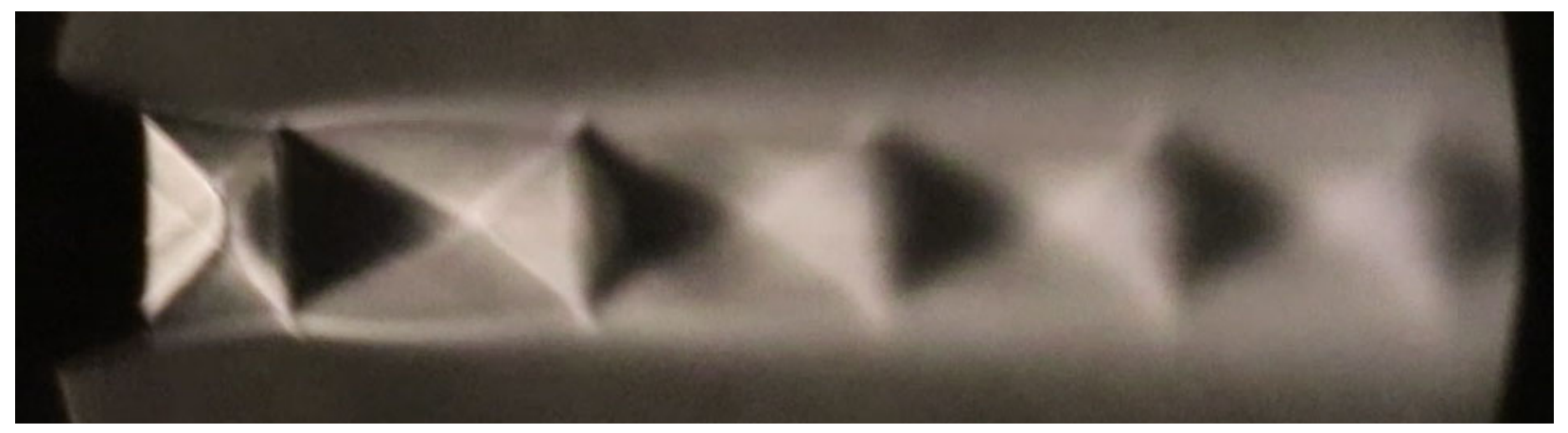

Fig. 2 Schlieren visualization of $M_{d}=2.0$ nozzle run over-expanded at a nozzle pressure ratio $r=4.2$.

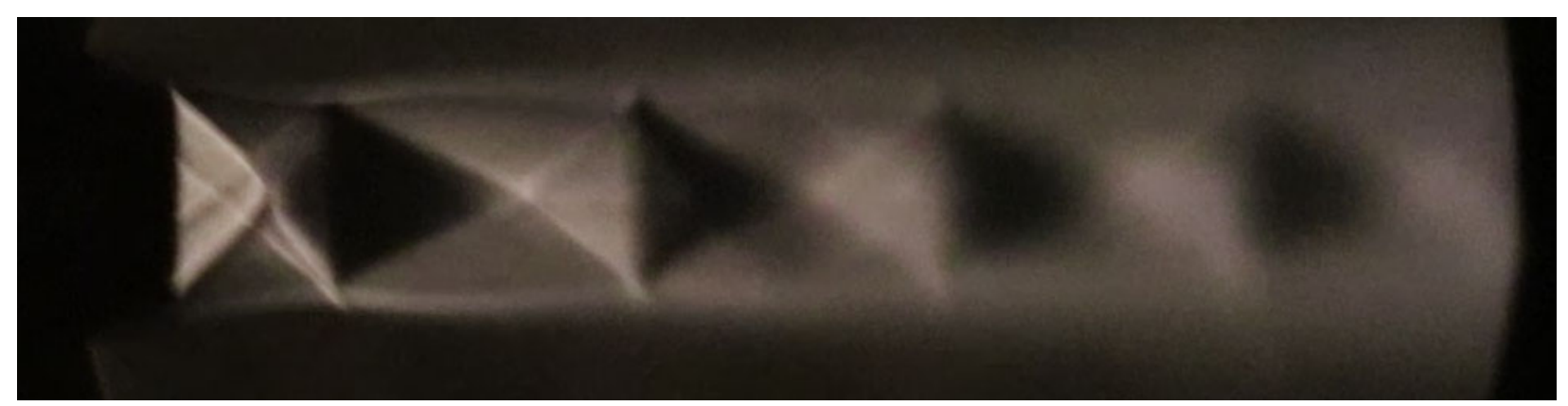

Fig. 3 Schlieren visualization of $M_{d}=2.0$ nozzle run over-expanded at a nozzle pressure ratio $r=4.7$.

pressure ratio is increased further to $r=5.75$, closer to the nozzle design pressure ratio $r=7.8$, then the strength of the expansions and of the compressions reduce, since the pressure in the jet column requires less adjustment to reach the ambient pressure. This allows for more oblique waves that are axially longer along the shock-cell train, as shown in Fig. 4 .

The axial length of the shock cells and the strength of the compressions and expansions are directly related to the shock-associated noise component of the jet acoustic signature in the acoustic far-field. This relationship is provided by Tam and Tanna [9], who related the frequency of the $n^{\text {th }}$ spectral peak of broad-band shock-associated noise to the phase velocity $U_{c}$ of the instability waves of frequency $f_{n}$ in the shear layer that grows from the nozzle lip and to the shock cell wavenumber $k_{n}$ as

$$
f_{n}=\frac{U_{c} k_{n}}{2 \pi\left(1-U_{c} / a_{\infty} \cos \theta\right)},
$$

where $a_{\infty}$ is the ambient speed of sound and $\theta$ is the direction of noise radiation measured from the jet axis. Tam et al. [10] state that $k_{n}$ is to be evaluated at the axial location at which the shear-layer instability waves reach their maximum

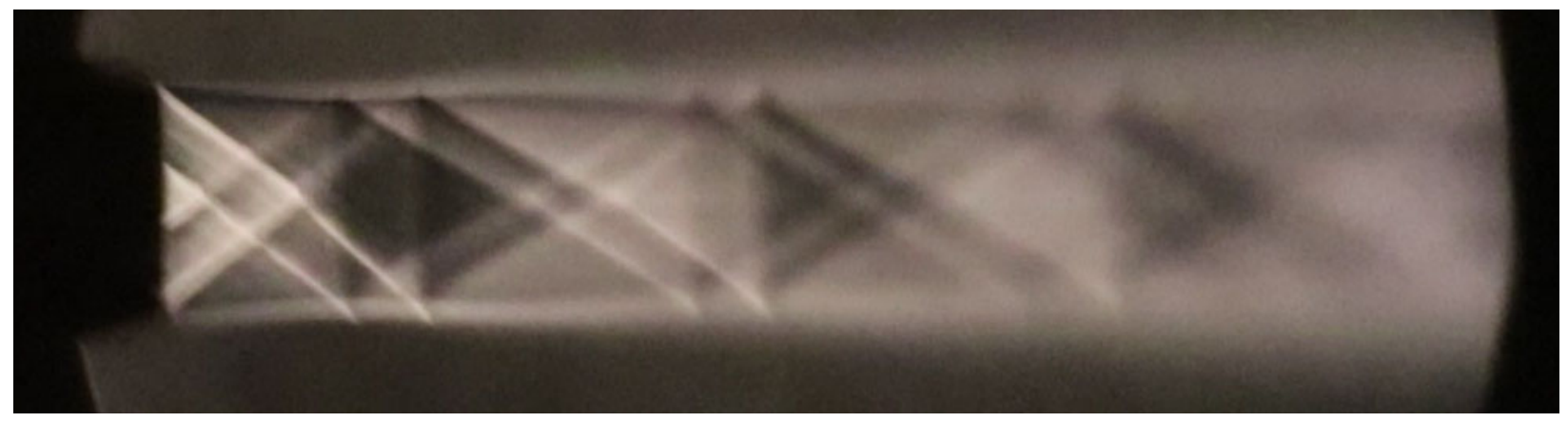

Fig. 4 Schlieren visualization of $M_{d}=2.0$ nozzle run over-expanded at a nozzle pressure ratio $r=5.75$. 


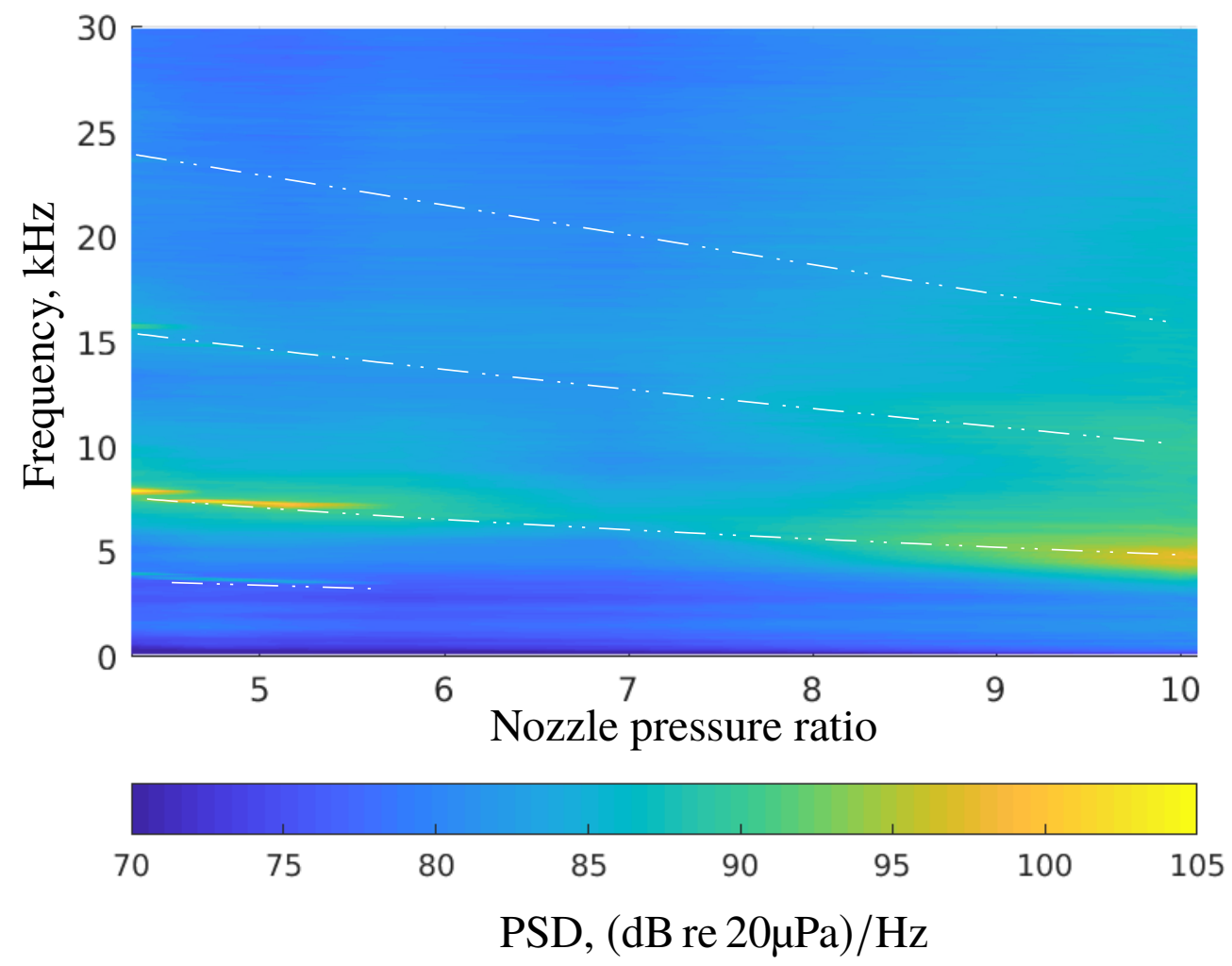

Fig. 5 Power Spectral Density $(\mathbf{P S D}$ in $(\mathrm{dB}$ re $20 \mu \mathrm{Pa}) / \mathrm{Hz})$ at different nozzle pressure ratios, measured at $80^{\circ}$ from the jet axis.

amplitude. Still, in general, as the shock cell spacing $\lambda$ is related to the shock cell wavenumber $k_{n}$ by $\lambda=2 \pi k_{n}^{-1}$, then the progressive axial stretching of the shock cell train documented by the sequence of Figs. 22 4 implies, by Eq. (1), a progressive reduction in broad-band shock associated noise frequency with increasing nozzle pressure ratio $r$ towards the fully expanded configuration.

\section{B. Far-field noise}

Figure 5 provides evidence of the progressive reduction in broad-band shock associated noise with increasing pressure ratio, by displaying the variation of the spectral content of the far-field acoustic pressure fluctuation measured at $80^{\circ}$ from the jet axis, over the nozzle pressure ratio range $4.3 \leq r \leq 10$. The data is overlaid by trend lines that indicate a monotonic reduction in the spectral peak frequency with increasing nozzle pressure ratio $r$, for the first three main spectral peaks. This also suggests that the noise radiation at $80^{\circ}$ from the jet axis has a significant shock-associated noise component. This is further confirmed by the levels of these dominant contributions increasing either side of the fully expanded nozzle pressure ratio $r=7.8$, at which the jet is designed to produce a shock-free discharge, and thereby to exhibit a shock-cell noise component more suppressed than at other nozzle pressure ratios.

With a Mach 2.0 contoured nozzle, shock-associated noise most strongly contributes to the jet noise radiation in the forward arc, away from the jet axis [11]. This feature is captured in Fig.6, which shows the change in the spectrum recorded by the 10 microphones with the microphone angle, at a constant nozzle pressure ratio $r=4.3$. At lower polar angles, over the range $20^{\circ} \leq \theta \leq 50^{\circ}$, the spectrum is characterized by a distinct low-frequency broad-band contribution, which varies in intensity with polar angle but the frequency distribution of which is substantially invariant with polar angle. This broadband contribution exhibits a broad peak at approximately $2 \mathrm{kHz}$ and a tone at approximately $7 \mathrm{kHz}$. This broadband contribution is associated to jet mixing noise. At higher polar angles, over the range $60^{\circ} \leq \theta \leq 80^{\circ}$, Fig. 6 exhibits an arc-shaped locus of elevated SPL running from $(\theta, f)\left(60^{\circ}, 22 \mathrm{kHz}\right)$ to $\left(80^{\circ}, 6 \mathrm{kHz}\right)$. This contribution, which narrows in frequency bandwidth with increasing polar angle, exhibits a broad peak that reduces in frequency 


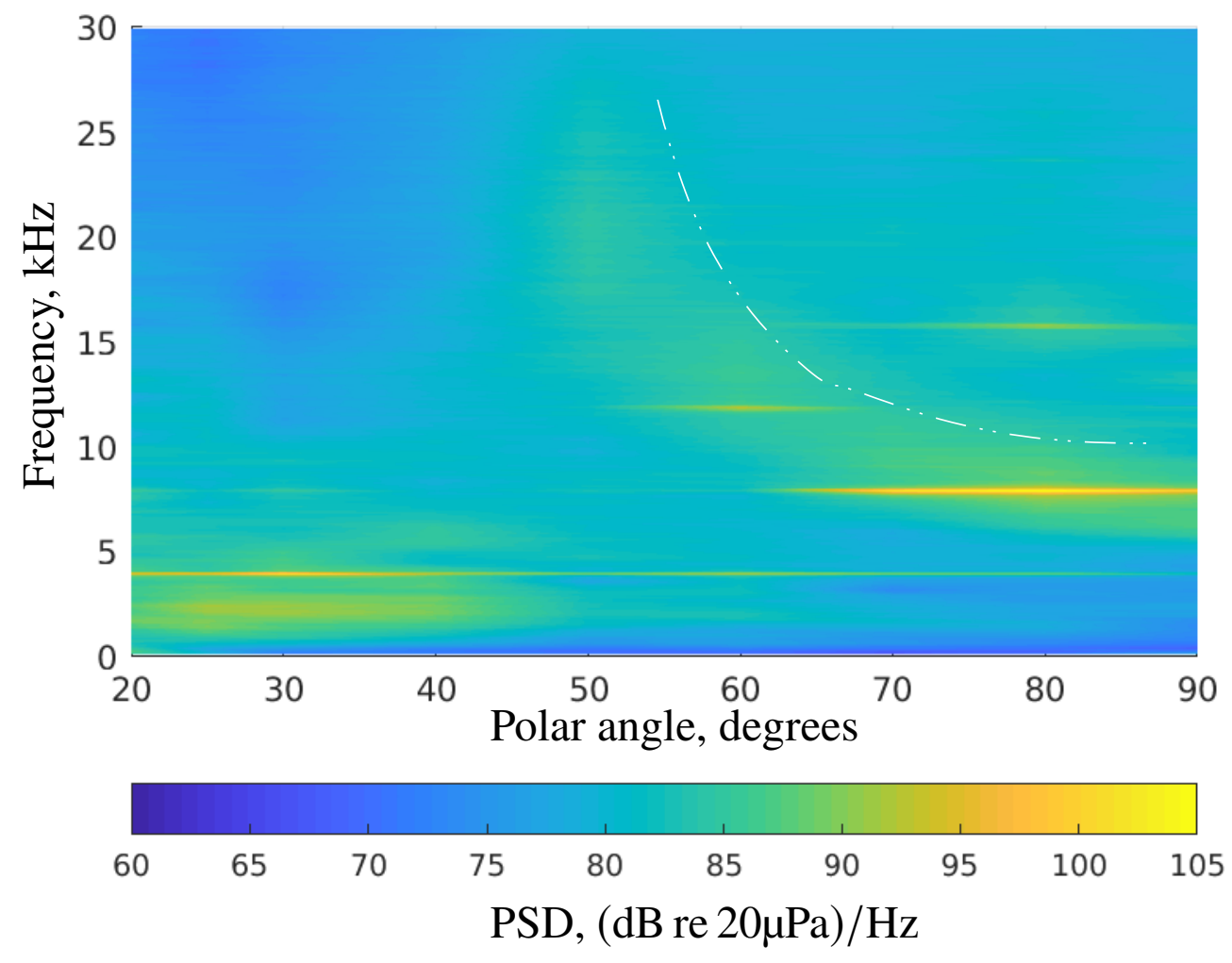

Fig. 6 Power Spectral Density (PSD in $(\mathrm{dB}$ re $20 \mu \mathrm{Pa}) / \mathrm{Hz}$ ) at different polar angles from the jet axis (nozzle outflow direction), measured at a nozzle pressure ratio of $r=4.3$.

with increasing polar angle. This bandwidth and peak dependence with polar angle is a characteristic of broad-band shock-associated noise, which is reported for incorrectly expanded Mach 2 nozzle tests by Norum and Seiner [12]. In Tam et al. [10], the narrowing of the bandwidth is related to the narrowing of the velocity spread of the shear-layer instability waves $\Delta U_{c}$ that, according to Eq. (1), are integral parts of the shock-associated noise production mechanism.

According to Tam et al. [10], a particular form of shock-associated noise is obtained for $\theta \rightarrow 180^{\circ}$ in Eq. (1), which leads to a self-excited feed-back loop between downstream-propagating shear-layer instabilities and upstream-propagating pressure waves. This feed-back loop, according to Powell [5], selects specific spectral bands for a noise emission that is referred to as screech tones, three of which are visible in Fig. 6 at about $6.5 \mathrm{kHz}, 9.75 \mathrm{kHz}$, and $13 \mathrm{kHz}$.

Therefore, in Fig. 6. two regions can be reasonably clearly identified, namely the region below a polar angle of $50^{\circ}$, where the spectrum exhibits recognizable characteristics of mixing noise, and above the $60^{\circ}$ polar angle, where the shock-associated noise contribution appears more dominant.

Operating the nozzle close to its design pressure ratio is shown in Fig. 7 to considerably reduce the arc-shaped signature of the broad-band shock-associated noise contribution over the range $60^{\circ} \leq \theta \leq 80^{\circ}$, although this is still noticeable as distinct from the dominant mixing noise contribution at lower polar angles. An interesting feature is observable at the polar angle of $40^{\circ}$. At this polar angle, the mixing noise contribution exhibits a frequency banded reinforcement over the frequency range $0.5 \mathrm{kHz}<f \leq 8 \mathrm{kHz}$.

This feature persists at the under-expanded jet regime shown in Fig. 8, at the same polar angle of $40^{\circ}$. At higher polar angles, the characteristic arc-shaped broad-band shock-associated noise contribution signature becomes again very noticeable in this under-expanded jet far-field sound pressure measurement.

Figures 68 indicate that the broad-band mixing noise contribution and the shock-associated noise contribution consistently dominate over different polar angle ranges across the tested range of nozzle pressure ratios, spanning between the over-expanded, the fully expanded, and the under-expanded regimes. This motivates the further analysis of the measured far-field sound pressure at the polar angles of $40^{\circ}$ and $80^{\circ}$, respectively, to investigate crackle and screech, 


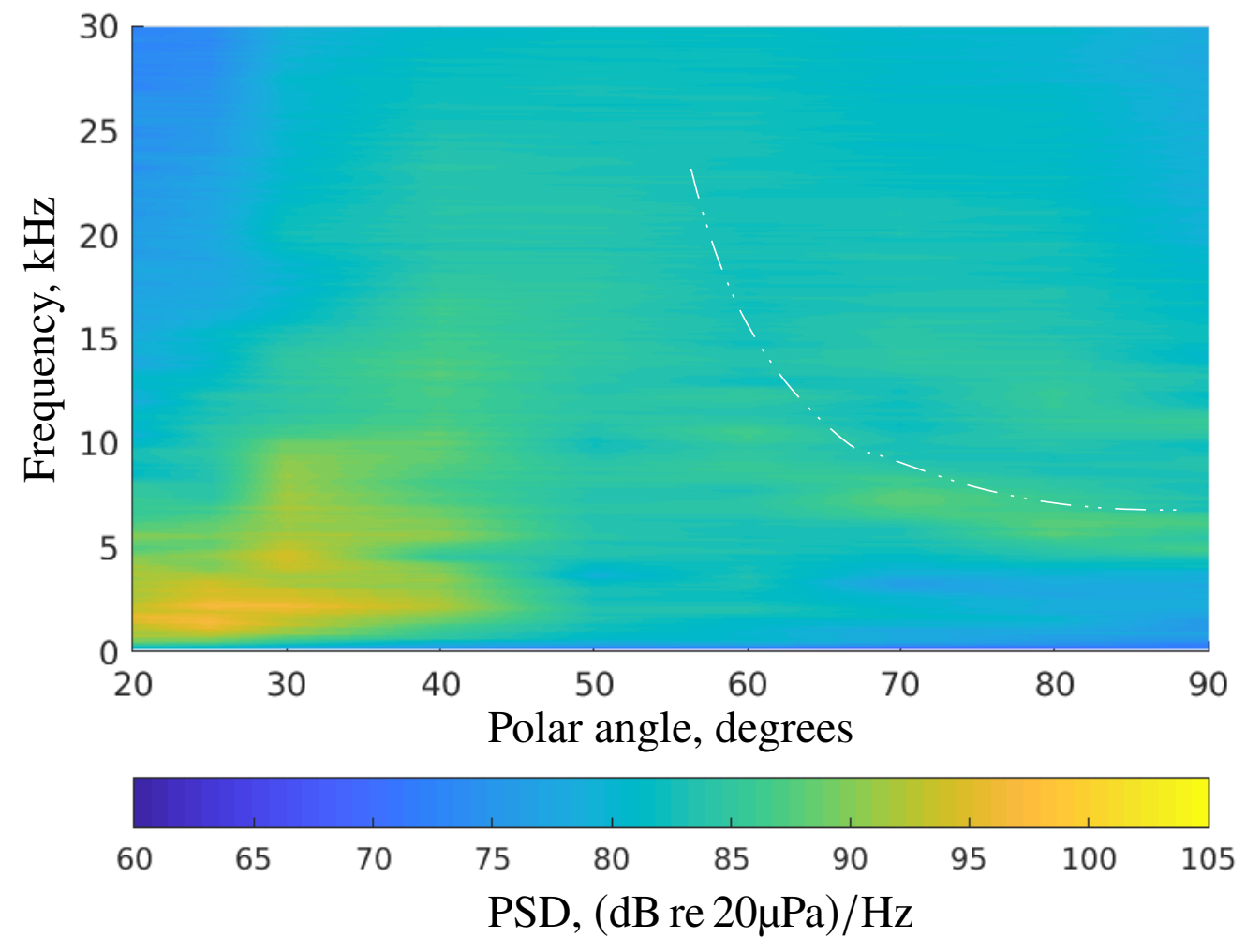

Fig. 7 Power Spectral Density (PSD in ( $\mathrm{dB}$ re $20 \mu \mathrm{Pa}) / \mathrm{Hz})$ at different polar angles, measured at a nozzle pressure ratio of $r=7.8$. 


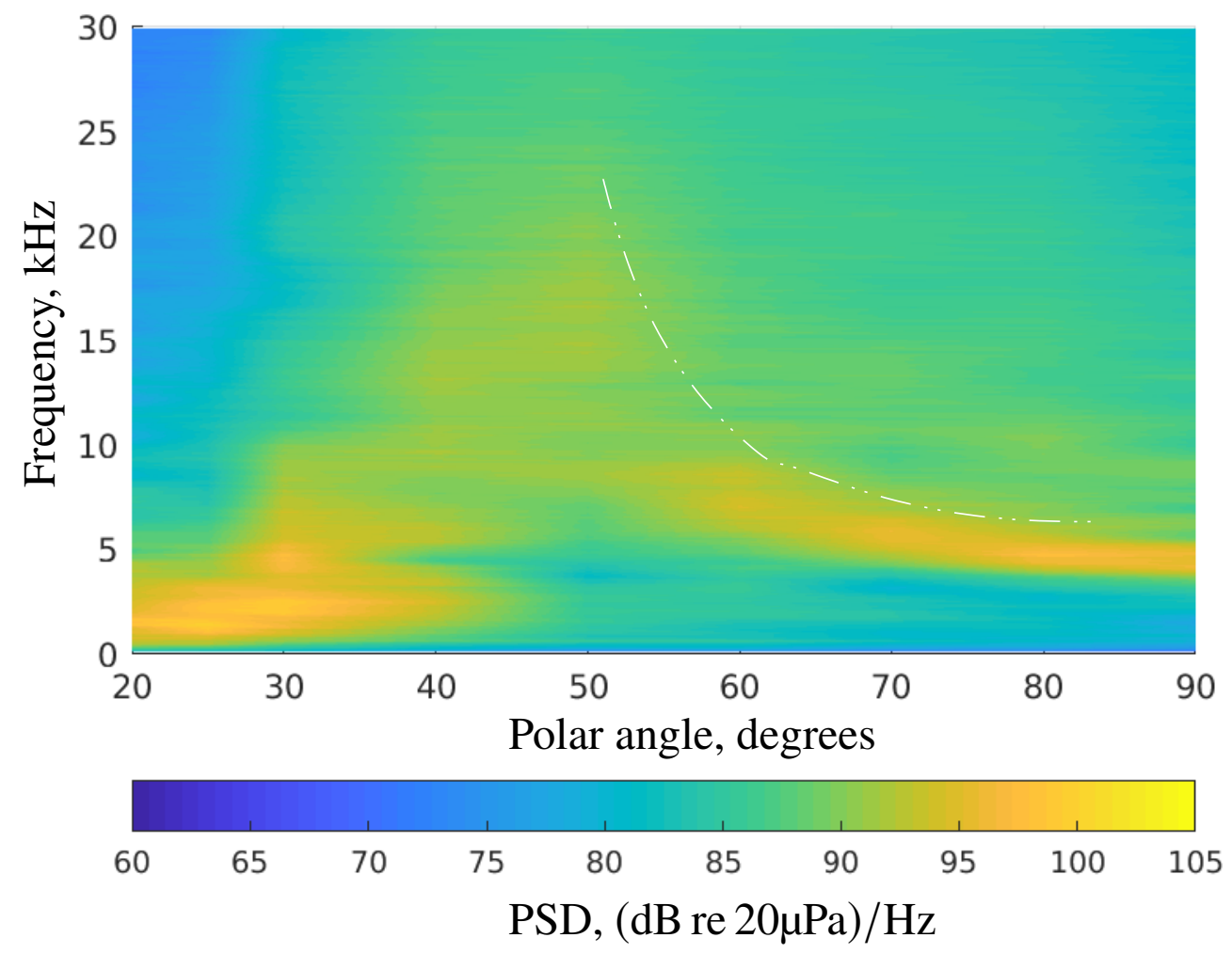

Fig. 8 Power Spectral Density (PSD in $(\mathrm{dB}$ re $20 \mu \mathrm{Pa}) / \mathrm{Hz}$ ) at different polar angles, measured at a nozzle pressure ratio of $r=10.09$. 


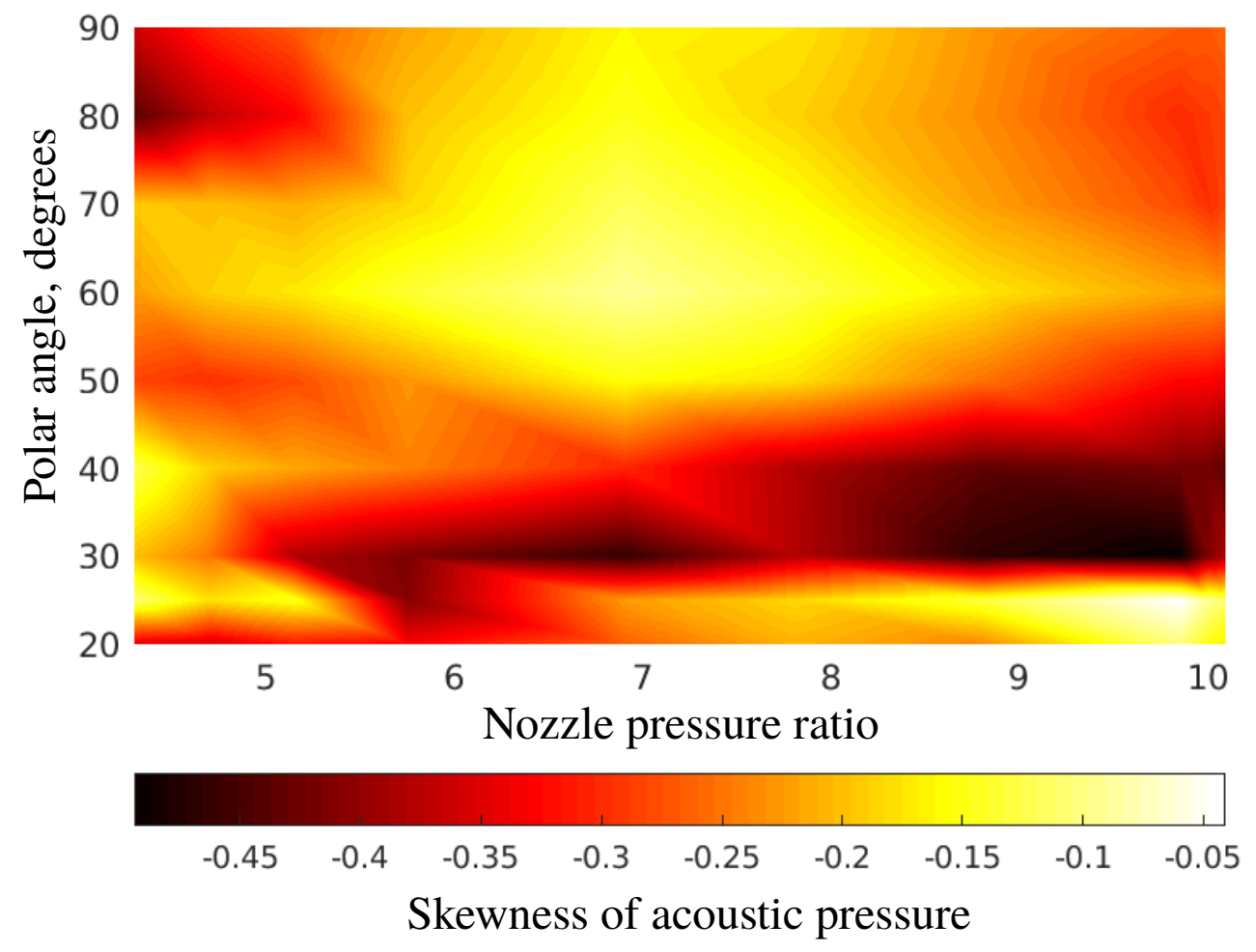

Fig. 9 Skewness of the acoustic pressure fluctuation at different polar angles and nozzle pressure ratios.

which are, respectively special features of broad-band mixing noise and of shock-associated noise. The rationale is that, even without the application of digital filters for suppressing screech for the analysis of crackle, and the converse, the observed dominance of shock-associated noise and of broad-band mixing noise at different polar angles may be sufficient for differentiating the characteristics of screech and crackle, by considering the acoustic pressure signals in the directions where shock-associated noise and broad-band mixing noise are respectively dominant.

\section{Skewness of acoustic pressure fluctuation}

Figure 9 shows the skewness of the frequency plot of the acoustic pressure fluctuation recorded by the acoustic array, over the nozzle pressure ratio range $4.3 \leq r \leq 10$. The darker regions in Fig. 9 denote combinations of radiation directions and nozzle pressure ratio at which noise has a statistically high magnitude of skewness. To interpret this result, it is recalled that random mixing, whilst it may be Gaussian as a noise source, it produces finite-amplitude waves towards the far-field. Non-linear propagation effects produce non-Gaussian far-field noise, which thereby has skewness $S_{k} \neq 0$. In this work, the threshold of $S_{k}=0.4$ is used to identify crackle in the far-field.

This threshold for crackle is selected based on the work by Ffowcs Williams et al. [2]. Ffowcs Williams et al. [2] studied the jet noise from the Olympus 593 engine. They described crackle as startling impulsive bangs and a similar description of this noise component is given in Gee et al. [13]. This makes crackle distinct from ordinary broad-band mixing noise, from shock-associated noise, and from screech. Ffowcs Williams further described crackle as a jet noise pressure fluctuation having a skewness in excess of 0.4. On this basis, this study uses the threshold $\left|S_{k}\right|=0.4$ in the analysis of the skewness of the measured sound pressure.

The aerodynamic noise from operating the jet over-expanded, over the nozzle pressure ratio range $4.3 \leq r<7.8$, is characterized by comparatively lower levels of skewness than over the under-expanded range, below a polar angle of $50^{\circ}$, where Figures 6 [ 8 indicate that the broad-band mixing noise contribution is dominant. This suggests a lower incidence of crackle in over-expanded nozzle operations. At the higher polar angle of $80^{\circ},\left|S_{k}\right|>0.4$ indicates the presence of a different noise signature, which reduces with increasing nozzle pressure ratio. It is recalled that Fig. 5 reported at 
$80^{\circ}$ a progressive reduction in the screech tone amplitude with increasing nozzle pressure ratio. This suggests that the observed high skewness level at $80^{\circ}$ is related to the amplitude of the screech tone, specifically it is thought that the large amplitude pressure waves associated to screech may generate a non-linear sound propagation with significant skewness [4].

In fact, at the fully expanded and at the under-expanded nozzle pressure ratios of $7.8 \leq r \leq 10$, over the polar angle range $60^{\circ} \leq \theta \leq 90^{\circ}$, Fig. 9 indicates relatively lower levels of skewness. These are noise radiation directions with a significant component of shock-associated noise. The amplitude of the narrow-band shock-associated noise progressively increases with increasing nozzle pressure ratio so that any screech tone remains embedded in the narrow-band shock-associated noise signature. The amplitude of the shock-associated noise component at the nozzle pressure ratio $r \sim 10$ is shown in Fig. 5 to be lower than that of screech at the over-expanded jet regime. This may have been sufficient for decreasing the non-linear sound propagation that is thought it is associated to screech and to acoustic pressure of high skewness.

Across the under-expanded nozzle pressure ratio range $7.8<r \leq 10$, at polar angles between $30^{\circ}$ and $40^{\circ}$, Fig. 9 shows the highest skewness values from the current measurements. This is a directivity range Figs. 6 8 associate to broad-band mixing noise, where the criterion of skewness $>0.4$ for the identification of crackle proposed by Ffowcs Williams is satisfied. There is therefore a strong indication of crackle being an identifiable component of broad-band mixing noise in under-expanded jets.

The results from the Mach 2 contoured nozzle indicate that, for under-expanded nozzle operations up to a nozzle pressure ratio of 10, the skewness of the sound pressure is sufficient for separating crackle from screech. For overexpanded nozzle operations over the pressure ratio range $4.3 \leq r<7.8$, skewness alone is insufficient for separating crackle from screech. Separation can be performed satisfactorily in over-expanded nozzle operations by combining skewness with directivity information, by noting that high values of skewness recorded at $80^{\circ}$ to the nozzle axis are associated to screech, whereas high values of skewness recorded at lower angles, between $30^{\circ}$ and $40^{\circ}$, are associated to crackle.

It is also of interest to note that, based on the skewness information of Fig. 99 at the over-expanded regime, screech and crackle appear to be mutually exclusive, or at least antagonists, in the Mach 2 nozzle acoustic far-field signature.

Accordingly to Kraichnan [14] and to Gee and Sparrow [15], non-linearly propagating finite-amplitude waves should have higher skewness in their gradients. This assertion is broadly confirmed by the current study, in which the first time derivative of the acoustic pressure $d p / d t$ is evaluated numerically from the sampled microphone records, from which its skewness $S_{k}(d p / d t)$ is determined. The results are reported in Fig. 10 .

At the under-expanded regime of $7.8<r \leq 10$, Fig. 10 helps to locate the maximum in the directivity of crackle at the $30^{\circ}$ angle. Computing the gradient of the acoustic pressure produces a greater difference in the value of skewness at this angle than at higher polar angles, which suggests that the radiation at $30^{\circ}$ is substantially more non-linear than at higher polar angles. This is consistent with the description of crackle reported in this work and with the absence of screech tones well above the broad-band shock associate noise, in this under-expanded regime.

At the over-expanded regime of $4.3 \leq r<7.8$, Fig. 10 confirms high skewness values at the high polar angle of $80^{\circ}$, in agreement with Fig. 9, where high-amplitude screech is also reported. This finite-amplitude propagation, with non-linear characteristics, would be consistent with the numerically higher value of $S_{k}(d p / d t)$ than $S_{k}(p)$ reported at $r=4.3$ and $\theta=80^{\circ}$. Contrary to Fig. 9. Fig. 10 reports high values of skewness at $\theta=25^{\circ}$ at $r=4.7$. The origin of this feature remains at present unexplained.

\section{Conclusion}

Collaborative work in high-speed jet noise has exposed fundamental aspects of the noise signature from model jets, at nozzle pressure ratios representative of the ones for propulsion systems of high-speed jet aircraft and of future supersonic civil transport aircraft. At these high operating pressure ratios, screech and crackle are observed.

Experimental measurements have exposed the usefulness of skewness as a characterizing parameter for identifying crackle in the far-field noise radiation. It is shown that the skewness of the sampled pressure fluctuation is best used in conjunction with the skewness of the time derivative of the sampled pressure fluctuation to characterize crackle. This appears to give better grounds for distinguishing crackle from screech, which can have a high third moment value in the pressure but has a comparatively lower third moment value in the pressure time derivative.

The measurements also expose that crackle and screech are either competitive sound generation processes or are mutually exclusive, in over-expanded jets, which, to the authors' best knowledge, is the first evidence of such occurrence documented in either numerical work or measurements on model jets or full engines. 

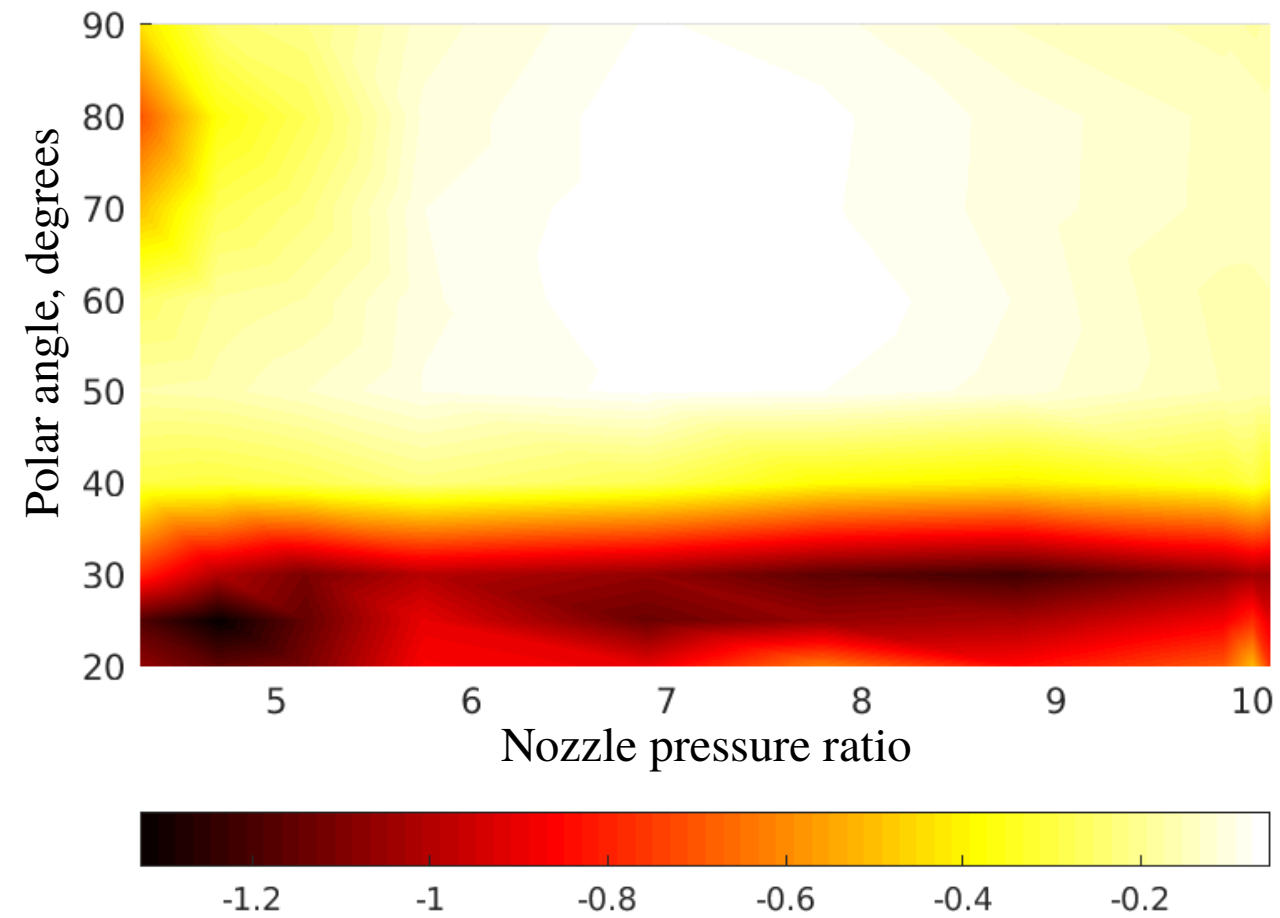

Skewness of the rate of change of acoustic pressure with time

Fig. 10 Skewness of the time derivative of the acoustic pressure fluctuation at different polar angles and nozzle pressure ratios. 
The greater understanding of the noise propagation and generation from high-speed jets this collaborative research is pursuing has good potential for benefiting the design and operation of high speed jet aircraft, reducing their adverse effect on military personnel, on the neighbourhood of airfields, and eventually enabling the operation of civil supersonic transport aircraft from existing civil airports near large cities.

\section{Acknowledgments}

This work was internally funded by Queen Mary University of London and by the University of Leicester, at which Punekar holds honorary positions. Collaboration amongst authors was supported by a Short Research Visit award by UK Fluids, under EPSRC grant EP/N032861/1. The technical support of Dr F. Motallebi for producing the schlieren records and of Mr P. D. Williams, for the signal acquisition hardware set-up, are gratefully acknowledged. The authors acknowledge early discussions with the late Prof. G. M. Lilley on screech and crackle, which have inspired them to undertake this research.

\section{References}

[1] Lighthill, M. J., "The inaugural Theodorsen lecture. Some aspects of the aeroacoustics of high speed jets," Theoretical and Computational Fluid Dynamics, Vol. 6, No. 5-6, 1994, pp. 261-280.

[2] Ffowcs Williams, J. E., Simson, J. E., and Virchis, V. J., “Crackle: An annoying component of jet noise,” Journal of Fluid Mechanics, Vol. 71, No. 2, 1975, pp. 251-271.

[3] Punekar, J. N., Avital, E., and Li, X., "Crackle: Measurements and analysis of supersonic jet noise," Asian Joint Conference on Propulsion and Power, 2012, pp. 1-9.

[4] Punekar, J. N., Avital, E., and Li, X., "Experimental investigation of nonlinear properties of crackle and screech in supersonic jets," The Journal of the Acoustical Society of America, Vol. 141, No. 6, 2017, pp. 1-7.

[5] Powell, A., "On the mechanism of choked jet noise," Proceedings of the Physical Society, Vol. 66, 1953, pp. $1039-1056$.

[6] Rona, A., "Aerodynamic and aeroacoustic estimations of oscillatory supersonic flows," Ph.D. thesis, Department of Aeronautics and Astronautics, University of Southampton, UK, Jun. 1997.

[7] Rona, A., and Zhang, X., "Time accurate numerical study of turbulent supersonic jets," Journal of Sound and Vibration, Vol. 270, No. 1-2, 2004, pp. 297-321.

[8] Seiner, J., and Norum, T., "Aerodynamic aspects of shock containing jet plumes," AIAA 6th Aeroacoustics Conference, Hartford, Connecticut, AIAA-80-0965, Vol. 1, AIAA, Washington, DC, 1980, pp. 1-17.

[9] Tam, C. K. W., and Tanna, H. K., "Shock associated noise of supersonic jets from convergent-divergent nozzles," Journal of Sound and Vibration, Vol. 81, No. 3, 1982, pp. 337-358.

[10] Tam, C. K. W., Seiner, J. M., and Yu, J. C., "On the relationship between broadband shock associated noise and screech tones," 9th Aeroacoustics Conference, Williamsburg, Virginia, AIAA 84-2276, Vol. 1, AIAA, Washington, DC, 1984, pp. 1-8.

[11] Seiner, J., and Norum, T., "Experiments of shock associated noise on supersonic jets," AIAA 12th Fluid and Plasma Dynamics Conference, Williamsburg, Virginia, 1979, pp. 1-13. 79-1526.

[12] Norum, T., and Seiner, J., "Measurements of mean static pressure and far-field acoustics of shock-containing supersonic jets," Technical Memorandum 84521, National Aeronautics and Space Administration, 1982.

[13] Gee, K. L., Tracianne, B. N., Wall, A. T., Downing, M. J., Micah, J., Michael, M., and McKinley, R., "Propagation of crackle-containing jet noise from high-performance engines," Noise control Engineering Journal, Vol. 64, No. 1, 2016 , pp. $1-12$.

[14] Kraichnan, R. H., "Models of Intermittency in Hydrodynamic Turbulence," Physical review letters, Vol. 65, No. 5, 1990, pp. 575-578.

[15] Gee, K., and Sparrow, W., "On the perception of crackle in high-amplitude jet noise," AIAA J., Vol. 45, No. 3, 2007 , pp. 593-598. 